\title{
Current paediatric orthopaedic practice in hereditary multiple osteochondromas of the forearm: a systematic review
}

\author{
Tamer A. EL-Sobky ${ }^{1, *}$, Shady Samir ${ }^{1}$, Ahmed Naeem Atiyya ${ }^{2}$, Shady Mahmoud ${ }^{1}$, Ahmad S. Aly ${ }^{1}$, \\ and Ramy Soliman ${ }^{2}$ \\ ${ }^{1}$ Division of Paediatric Orthopaedics, Department of Orthopaedic Surgery, Faculty of Medicine, Ain-Shams University, \\ Abbasia, Cairo, Egypt \\ ${ }^{2}$ Division of Hand Surgery, Department of Orthopaedic Surgery, Faculty of Medicine, Ain-Shams University, Abbasia, \\ Cairo, Egypt
}

Received 25 October 2017, Accepted 29 December 2017, Published online 21 March 2018

\begin{abstract}
Introduction: This systematic review aims to answer three research questions concerning the management of hereditary multiple osteochondromas of forearm in children: What is the best available evidence for the currently employed surgical procedures? What patient characteristics are associated with better prognosis? What disease characteristics are associated with better prognosis?

Methods: We searched the literature using three major databases with no publication date restrictions. To enhance search sensitivity and maintain precision we used keywords/subject terms correlating with patient population, problem and interventions. We used strict inclusion/exclusion criteria to improve validity evidence. Results: The search process yielded 34 eligible studies with a total of 282 patients (315 forearms). We comprehensively analysed study and patient demographics and interventions and outcomes. Eleven studies (32\%) had a long-term follow-up and 31 studies (91\%) were retrospective. Of the total number of forearms, ulnar lengthening $+/-$ associated procedures was used in 210 forearms $(66.7 \%)$, isolated osteochondroma excision in 65 forearms (20.6\%) and isolated distal radius hemiepiphysiodesis in 15 forearms (4.7\%) among others.

Discussion: Ulnar lengthening can restore radiologic anatomy, improve appearance and to a lesser extent objective clinical parameters like joint range of motion on the short/intermediate term. Isolated osteochondroma excision can relief pain and satisfy cosmetic concerns occasionally. There is poor evidence to suggest that surgery improves quality of life or function. Predictors of surgical success in regard to patient and disease characteristics remain elusive. Natural history and prospective randomized control studies where the control group receives no treatment should be rethought. They have the potential for bias control and identification of the ideal surgical candidate. The complex interplay between the confounding variables has undermined the capability of most studies to provide well-grounded evidence to support and generalize their conclusions. Valid quality of life scales should supplement objective outcome measures.
\end{abstract}

Key words: Children, Hereditary multiple exostoses, Multiple cartilaginous exostoses, Diaphyseal aclasis, Benign forearm tumours, Skeletal dysplasia, Ulna lengthening.

\section{Introduction}

Hereditary multiple osteochondromas (HMO) are uncommon benign bone tumors. They are usually diagnosed in early childhood. HMO are inherited in an autosomal dominant manner. The two genes in which pathogenic variants are known to cause HMO are EXT1 and EXT2 [1,2]. The pathogenesis is linked a critical reduction in heparan sulfate chain elongation [2]. Contrastingly, solitary osteochondromas are common benign

\footnotetext{
*Corresponding author: tamersh@hotmail.com
}

bone tumours. The growth pattern of a solitary osteochondromas is comparable to that of HMO. The diagnosis of $\mathrm{HMO}$ is based upon a distinct clinical and radiographic appearance. A fundamental clinical feature is multiple firm swellings erupting from the ends of long bones or from the surface of flat bones usually symmetrical [1]. Radiologically they present as multiple juxtaphyseal cartilage capped bony growths with undisturbed course of cortex and medullary bone from the normal bone into the osteochondroma [1,2]. Masada and colleagues [3] classified HMO into three main groups based upon the pathologic anatomy. Patients with HMO can exhibit limb 
length discrepancy, angular deformities around the knee, ankle and forearm, short stature, painful joint range of motion, joint subluxation and neurovascular compression $[1,2]$. Up to $70 \%$ of patients with HMO manifest in forearm deformities [4]. Most forearm and hand deformities are clinically pronounced $[4,5]$. Unbalanced physeal growth between the radius and ulna can result in forearm bowing, relative shortening of radius or ulna, carpal instability, and radial head dislocation with subsequent limitation of forearm rotation $[1,4]$. Forearm HMO has been managed by one or more of the following procedures: isolated excision of osteochondroma [6-12], acute $[7,12,13]$, and /or gradual [6,14-26] ulnar lengthening, combined ulnar and radial lengthening [27], distal radial hemiepiphysiodesis $[3,12,28,29]$, corrective radial osteotomy $[7,13,30]$, creation of one-bone forearm [31-33], radial head relocation $[8,10,22]$, reconstruction of the distal ulnar epiphysis by vascularized proximal fibula epiphysis [34] and SauveKapandji procedure [10]. Nevertheless, the optimal management of HMO of the forearm is greatly disputable. The best evidence for each of the practiced surgical procedures, the optimal timing for intervention and predictors for surgical success are alike unsettled disputes. Some authors cast fresh doubts about the value of surgery in regard to improving function $[7,35]$. This topic has not been critically appraised before in the literature. This systematic review aims to resolve the above-mentioned disputes. In consequence we formulated the following research questions relating to the management of HMO of forearm in children: 1) What is the best available evidence for surgical procedures used to manage HMO? 2) What patient characteristics are associated with better prognosis? 3) What disease characteristics are associated with better prognosis?

\section{Methods \\ Search approach}

This article does not contain any studies with human participants or animals performed by any of the authors. All authors shared in the study selection and data extraction process relating to the surgical management of HMO of the forearm in children. We conducted a search for English language publications before July 2017 employing the following electronic databases: PubMed, Google scholar and Embase. We checked the reference lists of the captured articles and review articles for additional eligible publications. We also screened articles that cited the captured articles. We discarded non-peer reviewed literature that was not published in scientific journals and secondary research such as review articles, letter to the editor and commentaries. We conducted the initial search on May 2017. We performed an additional search prior to manuscript submission to make certain the extracted literature is updated. To expand the recapture of relevant studies our search strategy comprised both keywords and index words in accordance with Medical Subject Headings.
We used Boolean operators properly to optimize search results quantitatively and qualitatively. The three main Boolean operators are AND, OR, and NOT. Boolean operators are used to narrow, broaden or restrict the search results. We aimed at avoiding biased inclusion terms. Hence, the selection of search terminology was subdivided according to (a) patient population, (b) problem and (c) intervention terms. We retrieved relevant studies using the following patient population and problem terms: children, paediatric, hereditary multiple osteochondromas, hereditary multiple exostoses, multiple cartilaginous exostoses, diaphyseal aclasis, forearm tumors, ulnar shortening. Additionally, we used the following intervention terms: osteochondroma excision, radius osteotomy, ulnar lengthening, distraction osteogenesis. We did not impose limiting terms with regard to study design types. The collected studies were excluded as follows; (a) descriptive studies reporting the clinical and/ or radiologic features, (b) studies reporting the natural history, (c) studies reporting on adults, (d) studies reporting exclusively on solitary osteochondromas, (e) studies reporting solely on pathologies other than HMO, (f) studies with follow-up < one year, (g) HMO managed in the context of malignant transformation and (f) studies reporting solely on HMO of the hand. We included prospective and retrospective studies. We also included case series and case reports. If studies were heterogenous for age population and pathologic disorder, only skeletally immature patients with HMO were selected. Disputes in regard to study selection were settled with face-to-face meetings. A schematic representation of the literature extraction process together with exclusions is provided (Figure 1).

\section{Quality appraisal instruments}

The reported items of this review were in concordance with the Preferred Reporting Items for Systematic Reviews and Meta-Analyses statement [36]. We used the systematic review critical appraisal worksheet from the University of Oxford Centre for Evidence-based Medicine www.cebm.net/critical-appraisal to check quality through all phases of this systematic review [37]. We identified factors that may reflect a significant research bias before, during and after the conduction of included studies with respect to patient selection, outcome measure assessment, statistics and confounding variables. We employed a valid instrument designed to evaluate the methodological quality of non-randomized surgical studies, whether comparative or non-comparative (MINORS) [38]. We selected MINORS [38] evaluation instrument as case series observational studies were the key of the primary studies. We conducted a comprehensive comparison between the aggregated treatment outcomes and tried to identify patient and disease features linked to better prognosis. To avoid bias in favour of reporting positive findings only we decided to finalize our systematic review protocol in advance of any data extraction. 
Chief reasons for article exclusions $(n=54)$ :

- Descriptive studies only $(n=12)$

- Natural history/predictive studies $(n=14)$

- Solitary osteochondroma $(n=6)$

- Disagreeing anatomic site/age $(n=1)$

- Language $(\mathrm{n}=18)$

- Irretrievable $(n=1)$

- Severely deficient information $(n=2)(5$ patients)
Electronic databases searched:

Pubmed, Google scholar, Embase

Hand search: reference lists of

collected articles/ review articles

Search terms: hereditary multiple

osteochondromas, hereditary

multiple exostoses, multiple

cartilaginous exostoses, diaphyseal

aclasis, forearm tumors, ulnar

shortening, osteochondroma

excision, radius osteotomy, ulnar

lengthening

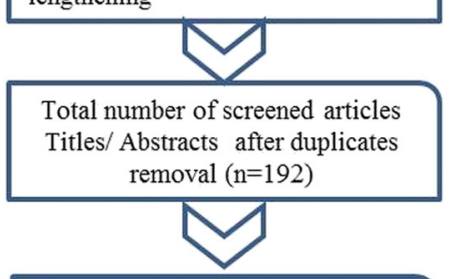

Number of full-text articles retrieved for eligibility assessment $(n=88)$

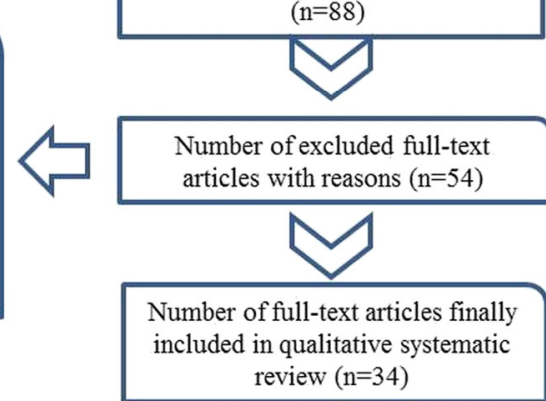

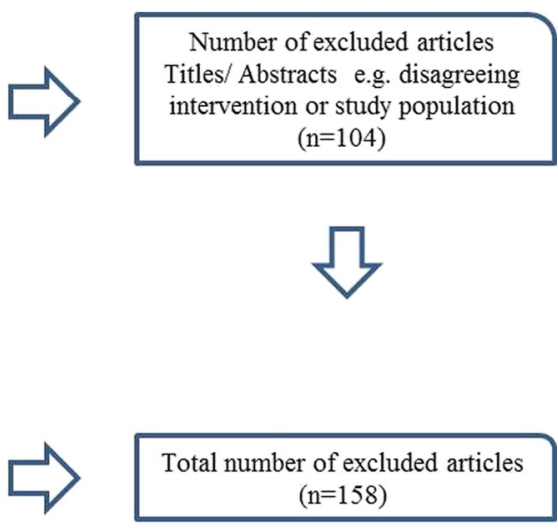

Figure 1. A schematic representation of literature extraction process.

\section{Results}

\section{Study demographics}

The literature extraction process involved 4 phases: (a) identification, (b) screening, (c) eligibility, and (d) inclusion. There were only three prospective case series studies $[20,27,39]$ (OCEBM type II) versus 31 retrospective studies (OCEBM type III). These prospective studies had small sample sizes [39] or were simply case reports $[20,27]$. The characteristics of the 34 final studies included in this systematic review are presented in Table 1. Eleven studies $(32 \%)$ had a follow-up $>5$ years, five of which were relatively sizable with study participants $\geq 10$. Of the 34 included studies, 32 were published in recognized orthopaedic society journals. The publication years ranged from 2016 to 1984 . Eight were multicentre studies $[8,11,13,15-$ 17,19,23]. According to MINORS the methodologic quality all but three prospective studies scored 5 out of 8 .

\section{Patient characteristics}

The summed number of patients enrolled in the included studies was 304 . One study with 22 patients was excluded from analysis due to poor demographic reporting but its implications were discussed [29]. Therefore, the final number of patients enrolled was 282 patients with 315 forearms. The mean age of patients for individual studies ranged from (513.5) years. One study provided a separate mean age for each of the three surgical procedures conducted [10] and another provided a separate mean age for boys and girls [28]. Gender distribution was provided for 222 patients (79\%) of the 282 patients finally enrolled in the review. There were 118 (53\%) males and 104 (47\%) females. Six studies did not provide the gender distribution $[7,10,11,16,28,29]$. One study did not provide a mean age or follow-up and provided a range instead [29]. For publications that did not directly report the Masada types the information was extrapolated from descriptive clinical picture and/or radiographs. Interestingly, in one study [26] neither Masada types nor osteochondroma excision were reported. Of the 257 forearms that "reported" Masada types, 166 (64.6\%) were type I, 25 (9.7\%) were type IIA, $48(18.7 \%)$ were type IIB and $18(7 \%)$ were type III. Two studies [22,25] referenced Masada but did not provide details of patients' radiographic grading.

\section{Interventions and outcomes}

A detailed reporting of the interventions, outcomes, and complications at final follow-up of the included studies in this review is presented (Table 2). The indications of 


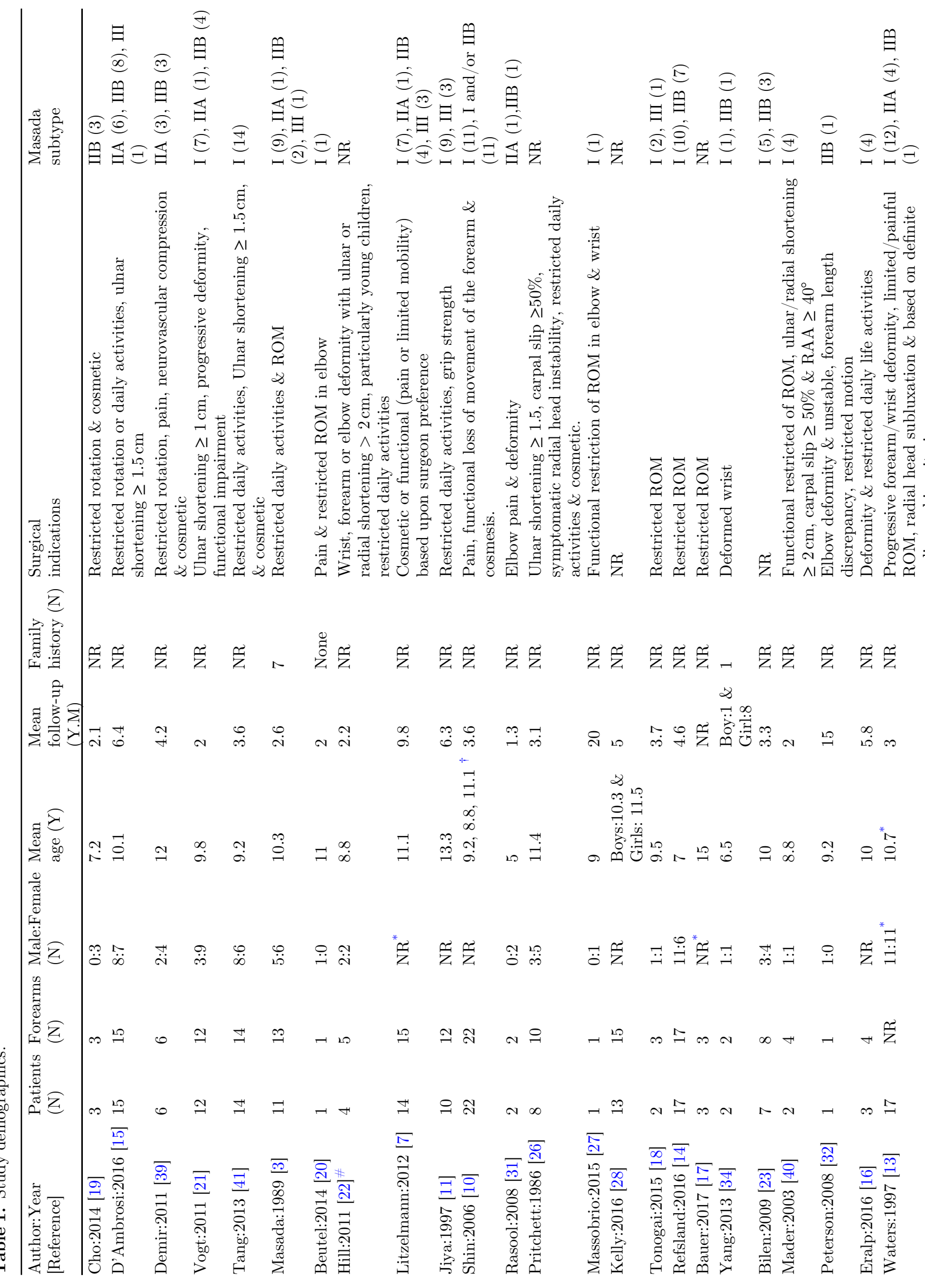


T.A. EL-Sobky et al.: SICOT-J 2018, 4, 10

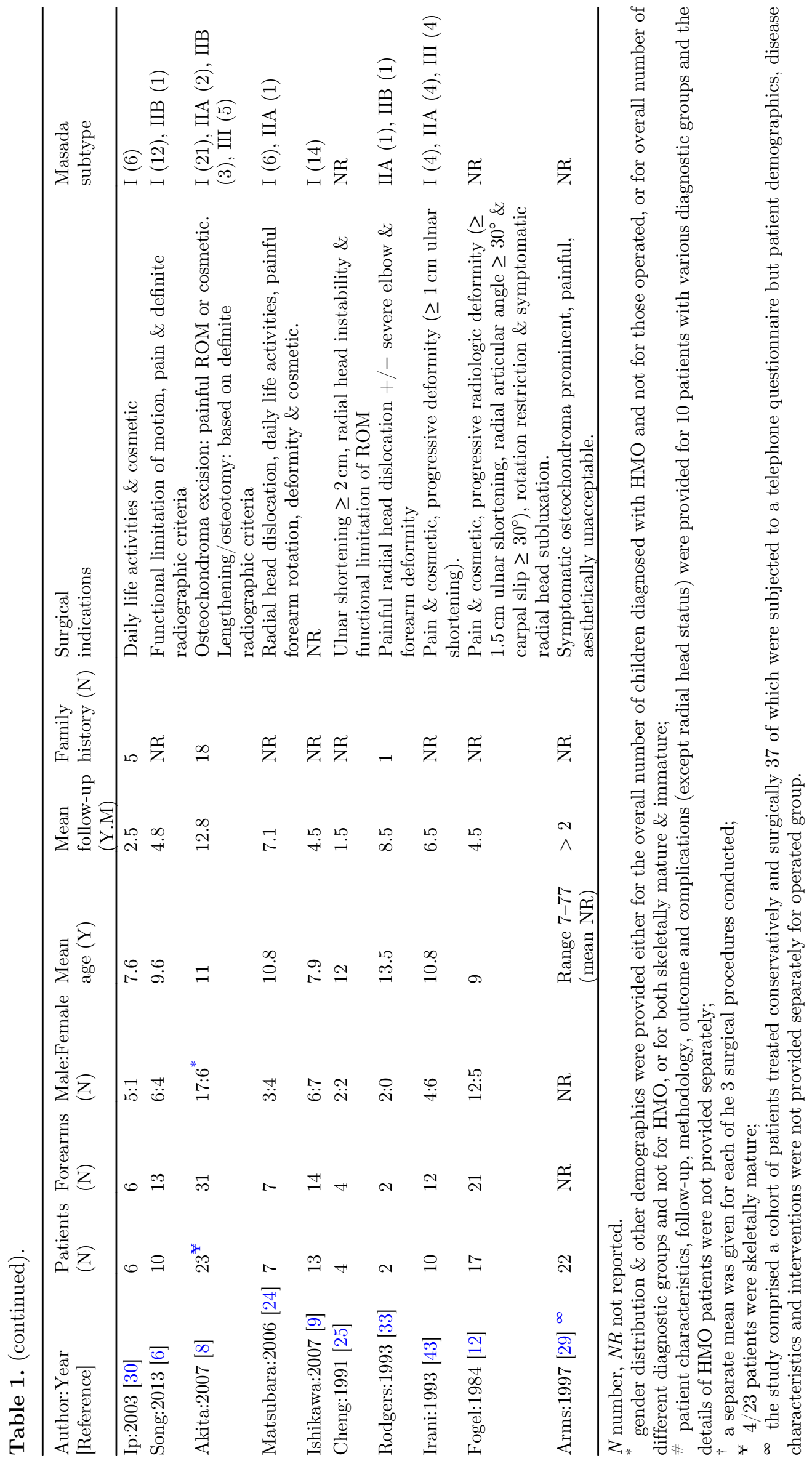




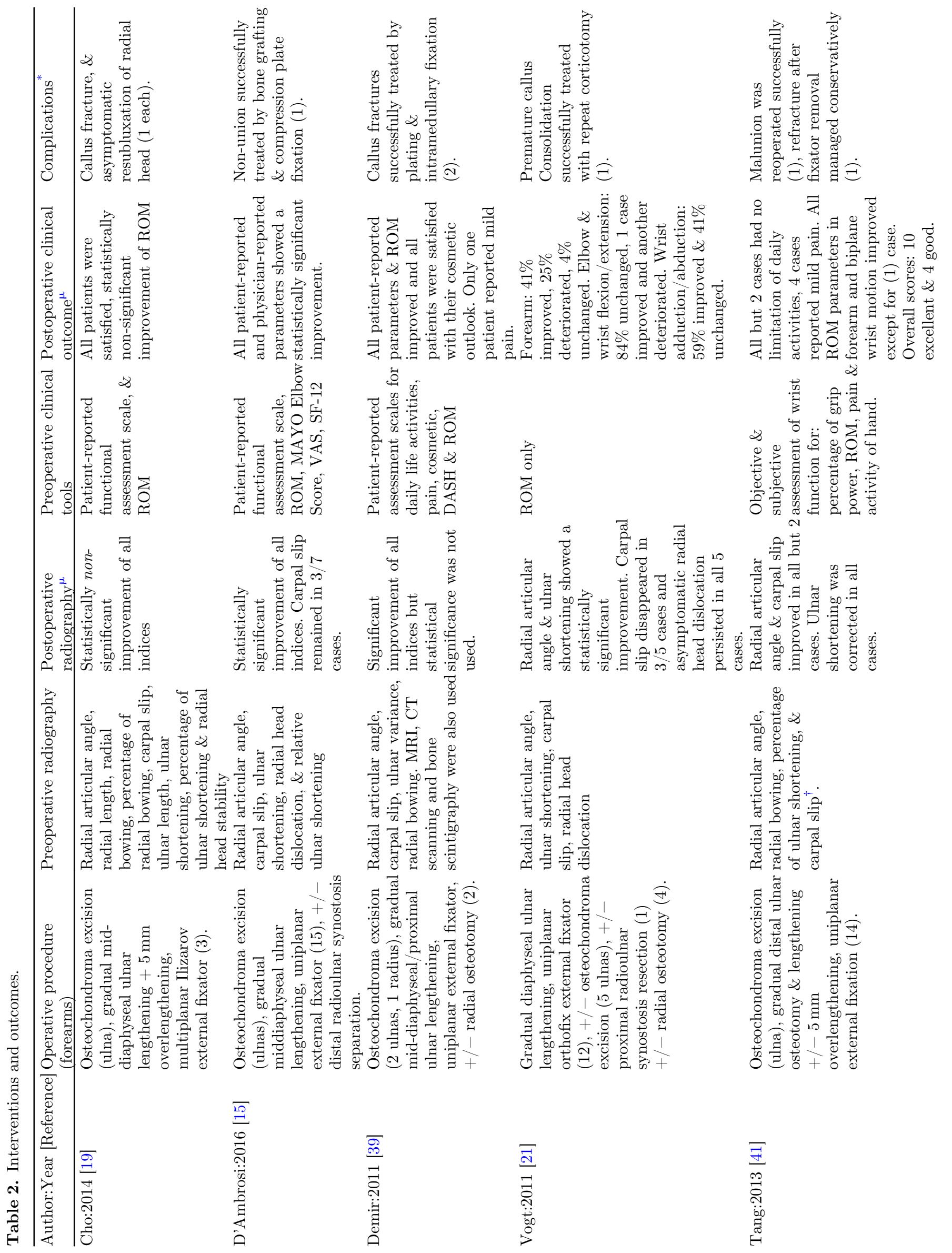




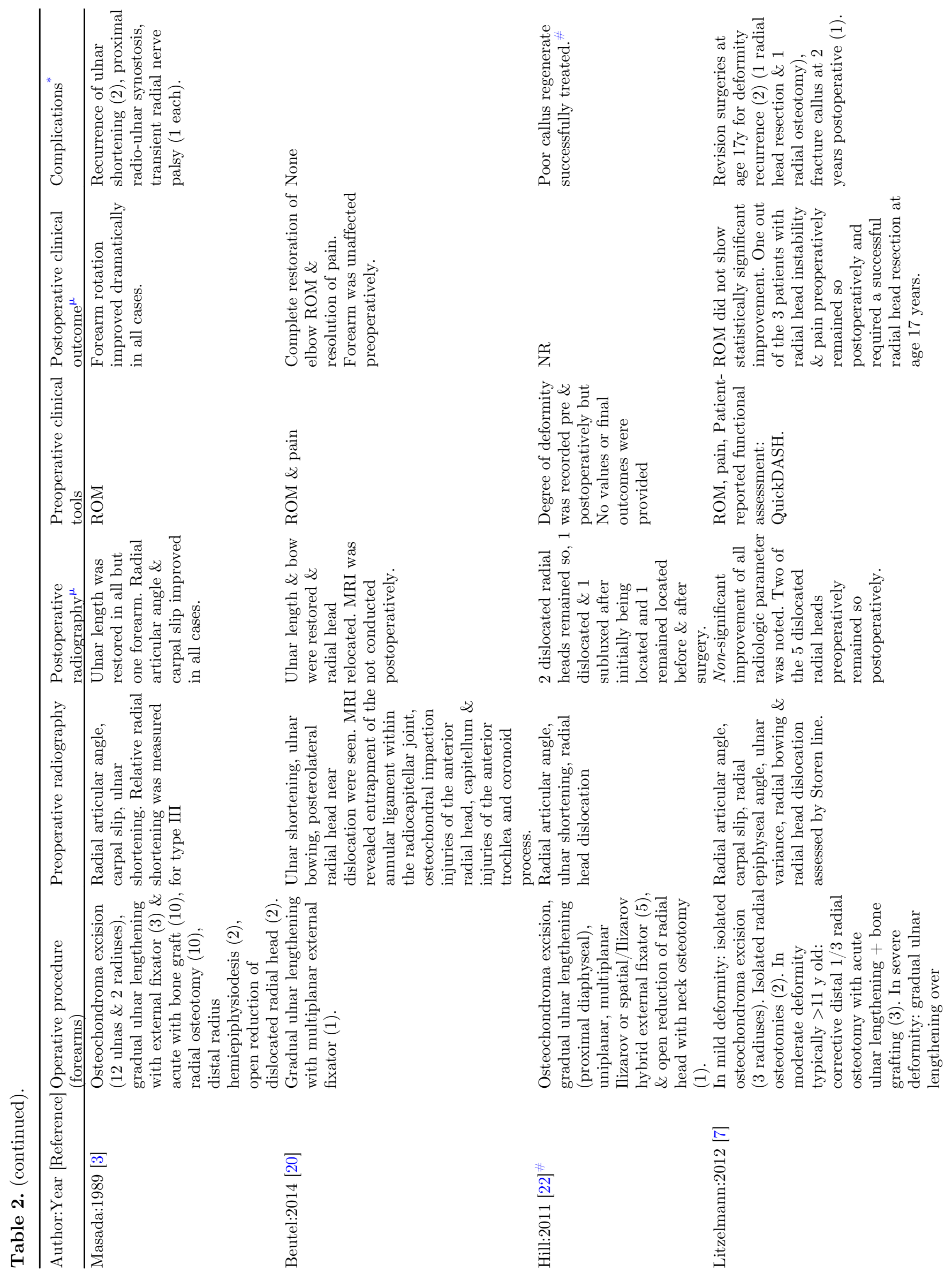




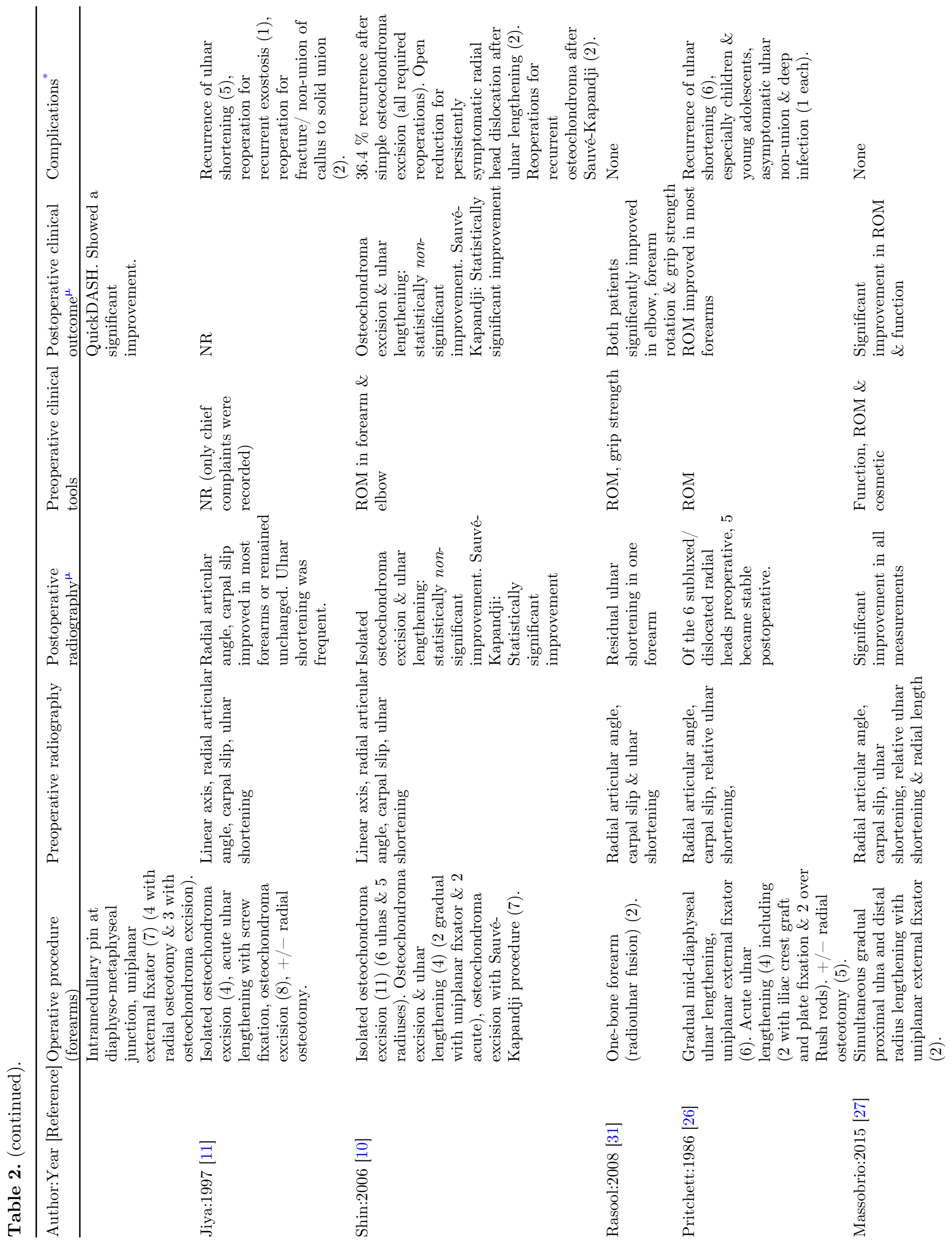




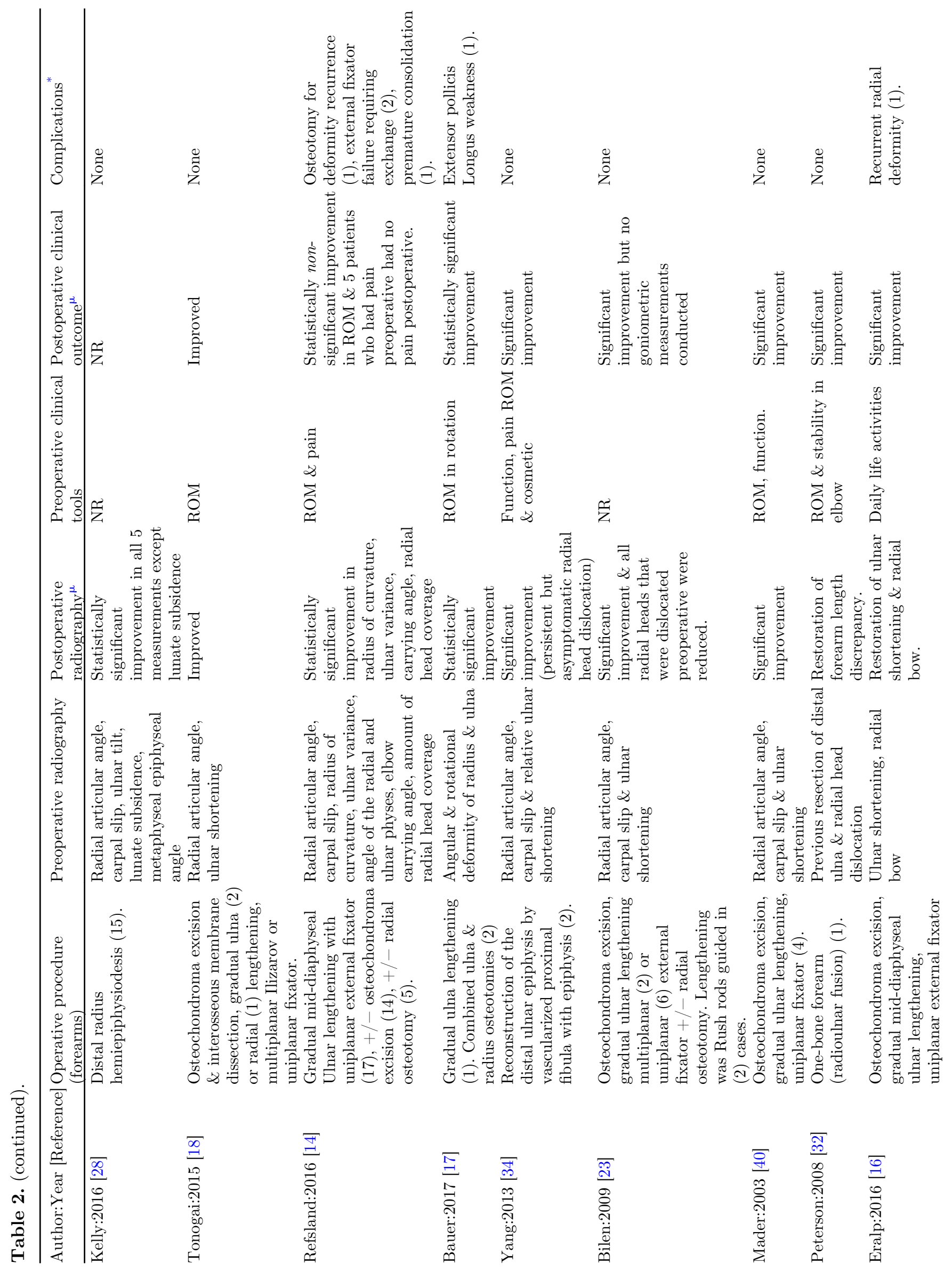




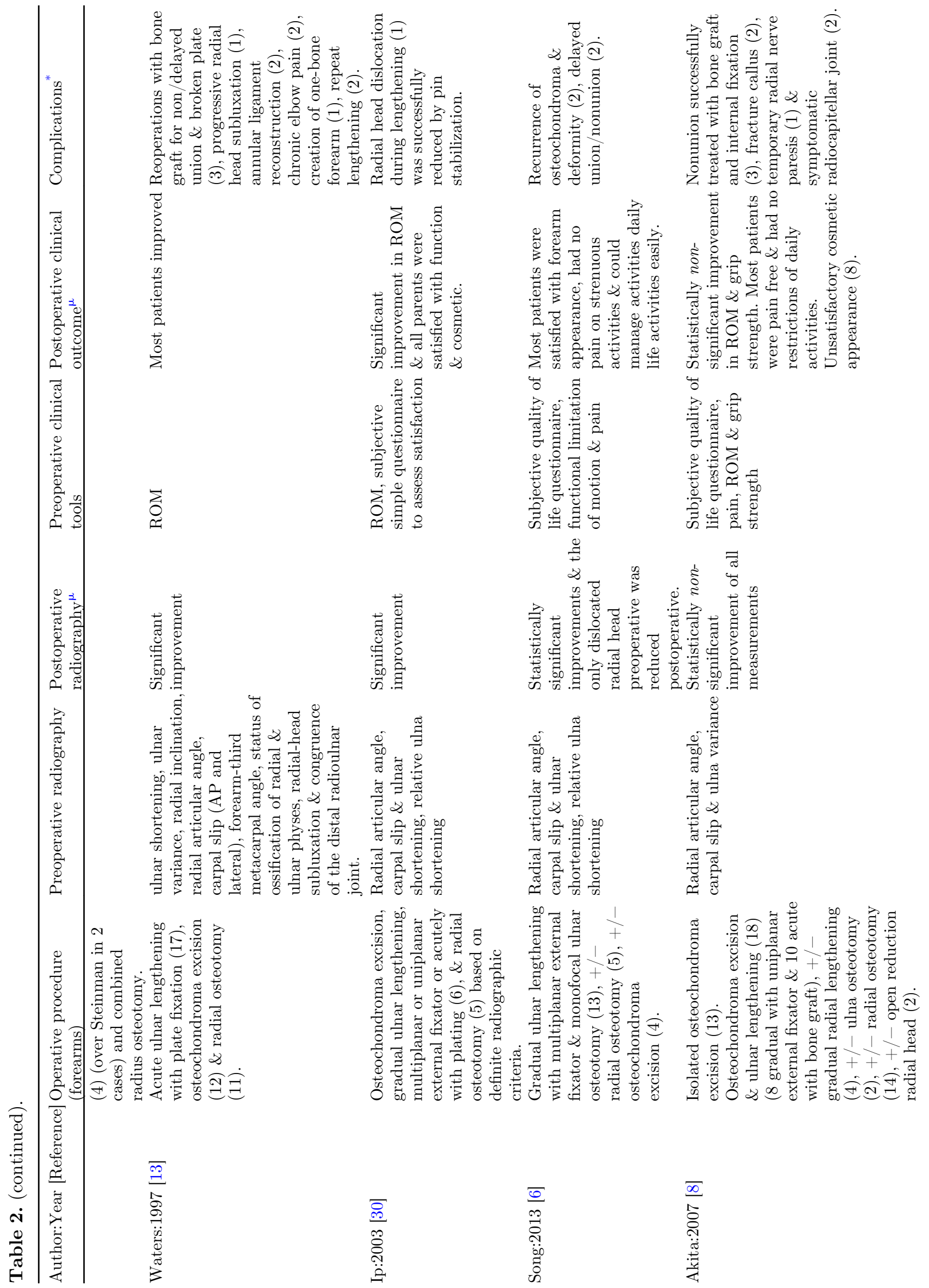




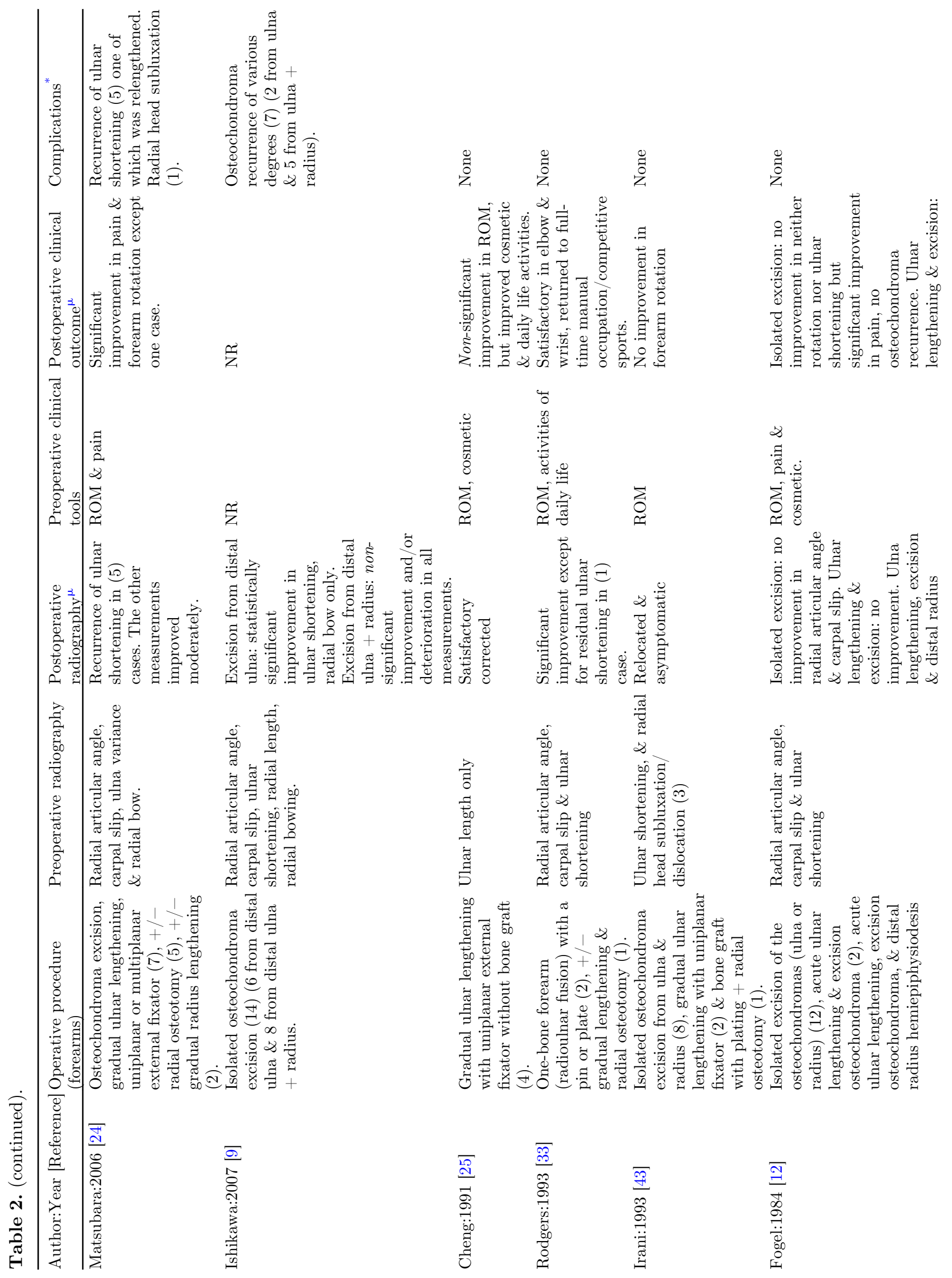




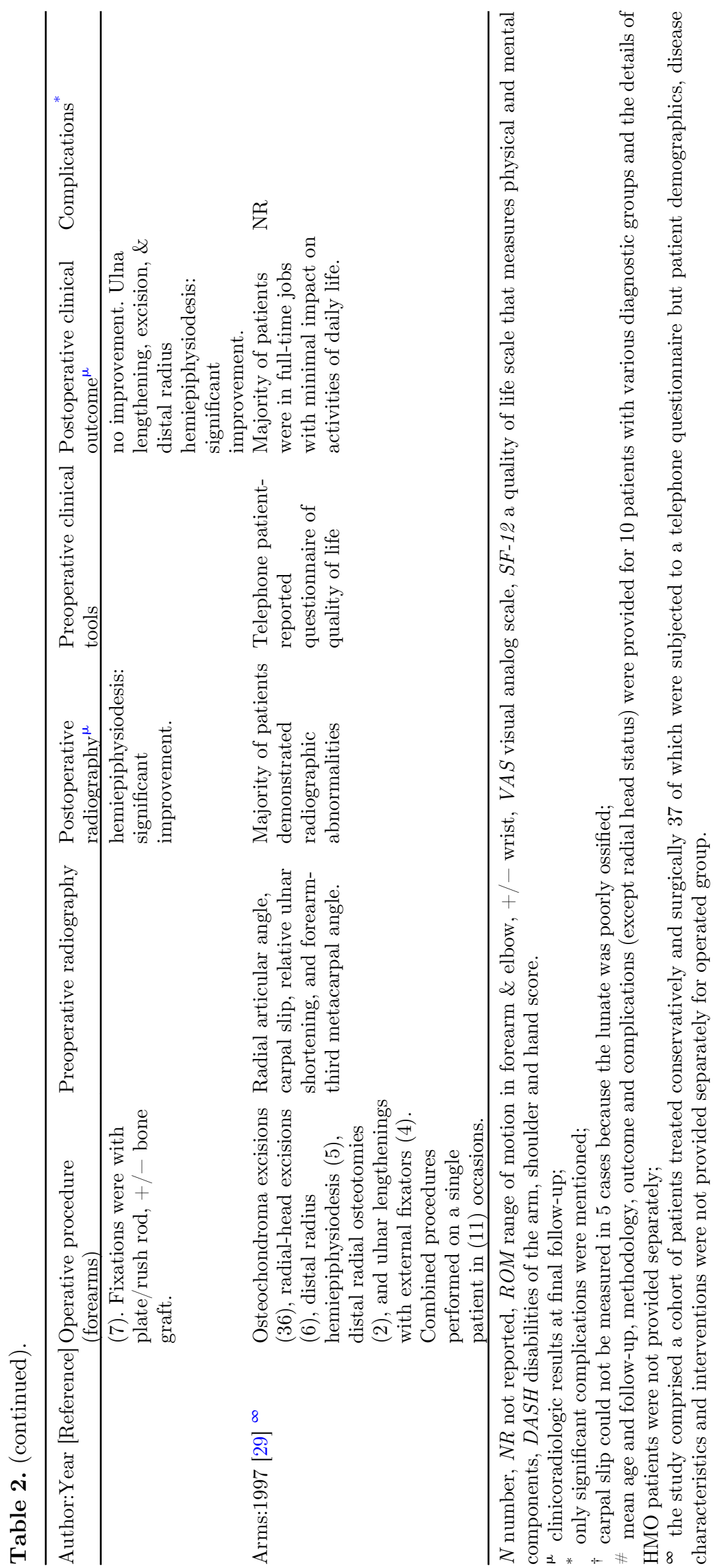


surgical intervention expressed variability among studies. Restriction of daily activities and/or range of motion in forearm/elbow were the most common indications of intervention. Most studies tended to favour objective physician-reported clinicoradiologic data as an outcome measure. Contrastingly, the majority of subjective patient-reported rating scales/questionnaires were nonvalidated and statistical significance was inadequately implemented. One study [7] restricted the implementation of the patient-reported rating scale on the sub-category of patients subjected to gradual ulnar lengthening by an external fixator. This is seen as an effort to reduce the impact of confounding variables. All studies employed plain radiography as a principal diagnostic tool. Two studies [20,39] employed MRI as an adjuvant imaging modality one of which [39] used CT scan and bone scintigraphy in addition. One study [27] employed ultrasound to monitor callus progression. The vast majority of studies assessed the radiographic outcome in accordance with proposed measurements by Fogel and colleagues which are a widely reported [12]. Five studies [6,9-11,41] used another referenced radiographic measurement by Burgess and Cates [42]. Among the various radiographic measurements, radial articular angle, carpal slip, ulnar shortening, and radial head stability were the most commonly shared by included studies. Contrastingly, radial length was the least used measurement. Ulnar lengthening $+/-$ an associated on demand procedure was conducted on 210 forearms $(66.7 \%)$ of the summed number of forearms. Associated procedures included a combination of one or more of the following: angular correction, osteochondroma excision, corrective radial osteotomy distal radial hemiepiphysiodesis and open reduction of radial head. Of the 210 forearms $(66.7 \%)$ subjected to ulnar lengthening, 145 forearms (69\%) were performed gradually and 65 forearms $(31 \%)$ were performed acutely mostly with bone grafting [3,7,8,1013,26,30]. Of these later studies two used acute ulnar lengthening exclusively [12,13]. All "gradual" ulna lengthenings were performed by a uniplanar fixator except in 17 forearms $(11.7 \%)$ a multiplanar fixator was used $[6,19,20]$ and five studies with 22 forearms (15.2\%) used both uniplanar and multiplanar [18,22-24,30]. All ulnar lengthening osteotomies/cortectomies were performed at a mid or proximal diaphyseal level. One study employed a simultaneous radial and ulnar lengthening in one forearm [27] and three others (seven forearms) conducted isolated radial lengthening as part of a series including ulnar lengthenings $[8,18,24]$. The vast majority of radial osteotomies were performed in association with ulnar lengthening procedures. Isolated corrective radial osteotomies were done in two forearms $(0.5 \%)$ only [7].

Isolated excision of osteochondroma or at least without bone lengthening was conducted on 65 forearms (20.6\%) of the summed number of forearms [7-12]. Temporary hemiepiphysiodesis of distal radial physis was performed on 29 forearms $(9.2 \%)[3,12,28,29]$ one of which [28] 15 forearms $(4.8 \%)$ was exclusively devoted to hemiepiphysiodesis. Sauvé-Kapandji procedure +/- osteochondroma excision was performed on seven forearms (2.2\%) [10]. Creation one-bone forearm through radioulnar fusion was practiced on five forearms (1.6\%) [31-33] and reconstruction of the distal ulnar epiphysis by vascularized proximal fibula including epiphysis was practiced on two forearms $(0.5 \%)$ [34]. An open reduction of radial head $+/-$ neck osteotomy was successfully performed on four forearms $(1.3 \%)[3,10]$ and unsuccessfully on three forearms $(1 \%)$ $[8,22]$. Resection of distal or proximal radioulnar synostosis was practiced on demand in two studies [15,21]. Radial head excision was not practiced in pediatric patients at least as a primary procedure. Proximal radio-ulnar fusion was practiced on one forearm to manage symptomatic radial head subluxation [12]. The comparative prevalence of the main interventions and techniques used in this review is demonstrated (Figures 2A, B, C). The overall complication rate was tolerable and showed no specific predilection for any of the main interventions employed in this review.

\section{Missing data}

The included studies had missing data related to the following items: gender distribution (five studies) $[10,11,16,28,29]$, number of forearms (one study) [29] and Masada subtype (seven studies) [12,17,22,25, $26,28,29]$. The overall skeletal burden of HMO was reported in only four studies $[11,16,33,43]$ and detailed in one [27]. Hand dominance was reported in only four studies $[19,31,33,39]$ and 11 studies used pain as an outcome measure. Previous surgeries were reported in four studies $[13,16,27,32]$ and age of initial presentation in four $[20,27,32,33]$. Results of histopathologic examination were reported in six studies [16,20,24,27,34,41] and family history in six $[3,8,20,30,33,34]$. No study reported genetic testing and physiotherapy protocols were either poorly or not reported at all except for one study [22]. One study did not report the follow-up period as it was primarily designed to assess the utility of a computer simulation model [17]. One study reported patient ethnicity [28]. The study that practiced reconstruction of the distal ulnar epiphysis by vascularized proximal fibula did not report donor site morbidity [34]. One study reported conclusions that were discordant with the results [11]. The authors underscored the importance of ulnar lengthening in preventing progressive deformity and minimising functional disability despite reporting frequent deformity recurrence rates [11]. Grippingly, the authors employed neither objective nor subjective clinical outcome measures [11]. Generally speaking, we suggest that some of the missing data may have a potential impact on the validity of results and conclusions. For example failure to report the overall skeletal burden of HMO in terms of number and location and pain can influence the subjective patientreported quality of life assessment [44-47]. Pain that was grossly underreported by the primary studies of this review has been found to be a major drive for surgery and negatively influenced by surgery [47]. The incidence of a positive family history in patients with HMO has been 
(A)

Ulnar lengthening $+/$ - an associated on demand procedure

Isolated excision of osteochondroma(s)

Isolated hemiepiphysiodesis of distal radial physis

- Sauvé-Kapandji procedure +/- osteochondroma excision

Others

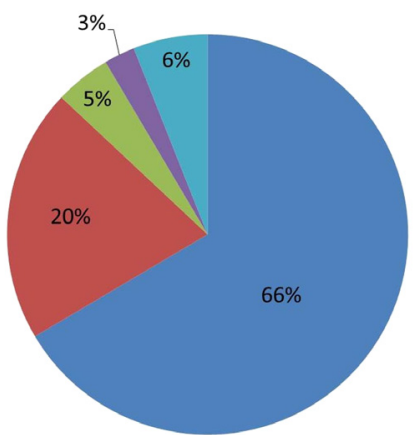

(B)
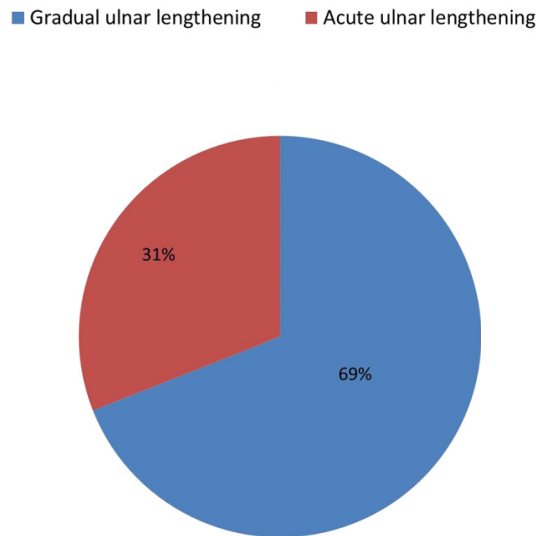

(C)
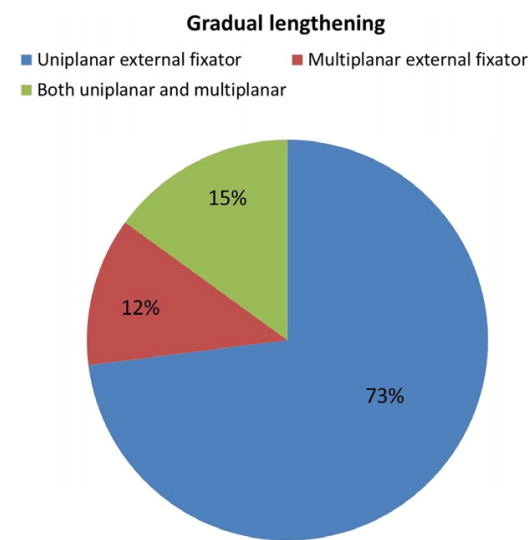

Figure 2. Comparative prevalence of the main interventions and techniques used in this review as percentages of forearms; (A) the main intervention groups used. Associated procedures include; osteochondroma excision, corrective radial osteotomy, distal radial hemiepiphysiodesis and open reduction of radial head. "Others" refers to isolated procedures as one-bone forearm, reconstruction of the distal ulnar epiphysis by vascularized proximal fibula epiphysis, radial osteotomy and lengthening; (B) gradual versus acute ulnar lengthening; (C) fixator choice of included studies.

estimated to range from 62-96\% [1]. Genotype-phenotype correlation studies demonstrated that certain types of gene mutations and the overall skeletal burden of HMO are associated with a worse clinical presentation particularly with respect to deformity and function $[1,2,44,45,48$ 50]. This may actually have a predetermined impact on treatment outcomes.

\section{Discussion}

\section{Summary of evidence}

This systematic review included many chief surgical interventions each of which was multifaceted. For clarity and consensus, we will discuss each chief intervention separately. We believe that this practical approach will assist in answering our first research question. The most prevalent combination of surgical procedures encountered in this review was ulnar lengthening, $+/-$ an associated procedure. Generally speaking, this surgical technique was adequately described and fairly constant across studies with a tolerable complication rate on the short-term. Nevertheless, there is poor evidence to demonstrate that the seemingly satisfactory results of many short-term studies are maintained on the intermediate-term [10] and more precisely on the long-term $[7,8]$. These long-term relatively sizable well-designed studies have questioned the value of surgical intervention even in association with deformity and radiographic abnormalities [7,8]. These studies correlated the clinicoradiologic outcomes with patients' self- reported functional outcome measures in children operated for HMO of forearm and argued for the choice of conservative treatment $[7,8]$. They found insufficient functional gains to justify surgery. In other words, preoperatively patients reported minimal func- tional impairment despite major clinical and radiologic abnormalities $[7,8]$. The only exception was symptomatic radial head dislocation. This discrepancy between the functional capacity and amount of forearm deformity was corroborated by three relatively sizable studies $[22,24,29]$. These findings have been confirmed by a natural history in a large cohort of untreated adult subjects with HMO [35].

Generally speaking, most studies tended to focus on the radiographic outcome measures at the expense of objective clinical parameters. Likewise, the objective physicianreported clinical outcome measures were implemented at the expense of the subjective patient-reported measures which were mostly non-validated and lacked in depth. Notwithstanding, these studies reported recognized cosmetic satisfaction of patients. Interestingly, in some studies patients were selected for surgery exclusively based upon radiographic criteria while clinical (objective or subjective) outcome measures were neither reported before nor after surgery $[9,11,22,28]$. It is noteworthy that complications such as recurrence of osteochondromas, and/or forearm deformity need longer durations to resurface especially in the skeletally immature population $[9,24]$. This undoubtedly overemphasizes the significance of conducting long-term follow-up studies and greatly undermines the quality of evidence extracted from such short-term studies. Additionally, this critically calls attention to the value of the validated subjective patient-reported overall quality of life scales. Using these scales has shown that HMO patients had lower scores compared to the general population $[46,48]$. We understand that a comprehensive assessment of patients' outcomes entails both subjective patient-reported and objective physician-reported instruments. In the light of such observations, it is important to rethink the cost/benefit profile of surgical intervention in paediatric HMO. In that regard, retrospective natural history studies may refine the 
wide and crude indications of surgery currently used in children with HMO. Two natural history studies are praised for aiming to identify radiographic predictors of radial head dislocation [4,51]. Likewise, well-designed randomized control studies where one group receives a definite surgical treatment and the other receives no treatment should be rethought. To satisfy the ethical demands of such studies, strict inclusion/exclusion criteria will have to be implemented before enrolment and randomization. Besides, adequate patient orientation in regard to risks and benefits of each treatment group and nature of the study will have to precede enrolment and randomization. Such study designs can generally yield valid results and generalizable conclusions. Nevertheless, we acknowledge the logistic and practical difficulties associated with such study designs. We believe that insufficient consensus about the indications of surgery and outcome scores in paediatric HMO is a major limitation of this systematic review. These discordant indications of surgery have also been noticed within individual study participants, further complicating evidence extraction. Likewise, the heterogeneity of the surgical procedures used, diversity in patient characteristics and relatively small study populations complicates evidence extraction. These above-mentioned features are inherent to retrospective studies that constituted the majority of included studies. Nonetheless, certain studies were remarkable for their methodologic quality. They implemented study designs that allow for control of selection and performance bias $[6,8,10]$. They neatly and separately reported the results of HMO patients in accordance with each distinct surgical procedure implemented. Additionally, some used assessor blinding methods and standardised surgeon related factors $[6,8,10]$. Isolated excision of osteochondroma can relief pain, satisfy cosmetic concerns and occasionally improve range of motion. Nevertheless, there is insufficient evidence for its use to initiate spontaneous deformity correction or improve overall limb function. In this review the cases of radial hemiepiphysiodesis, one-bone forearm, vascularized fibular graft, isolated radial osteotomies or lengthenings practiced as "stand alone" procedures were insufficiently prevalent to allow for meaningful conclusions. These procedures may be better suited to specific patient profiles and based upon surgeon's preference.

\section{Limitations and strengths}

We acknowledge limitations of this review. The majority of the included studies were of low methodologic quality. Studies with low methodologic quality may impact negatively on outcome validity and conclusions. Narrative/traditional reviews are usually biased and subjective in contrast to systematic reviews which are often unbiased and objective [52]. The reliability and accuracy of systematic review recommendations should not be determined by methodologic quality of the primary studies included, but rather by the degree of methodological integrity implemented by researchers [52]. In this systematic review we formulated focused research questions that require specific answers. Additionally, we implemented a comprehensive review methodology that allowed for a reasonable control of bias. Therefore, we estimate that our comprehensive systematic review strategy can counterbalance the shortcomings of including studies with low methodologic quality. Of the 18 studies excluded on basis of language 11 were provisionally eligible for inclusion in this review. These 11 studies comprised 54 forearms. It is noteworthy that in three of these 11 studies the size of study population could not be accounted for due to missing or deficient abstracts. In general, the excluded studies were comparable to the included studies in terms of methodology and individual sample size. Hence, these language exclusions seem inconsequential.

\section{Conclusions}

Ulnar lengthening $+/-$ associated procedures can restore radiologic anatomy, improve appearance and to a lesser extent objective clinical parameters on the short/ intermediate term. There is Poor evidence to demonstrate that these gains are maintained on the long-term. The impact of surgery on quality of life and function has not been adequately investigated. Considerable evidence suggests that surgery minimally impacts preoperative function. Predictors of surgical success in regard to patient and disease characteristics remain elusive. The complex interplay between the arrays of confounding variables has undermined the capability of most studies to provide well-grounded evidence to support and generalize their conclusions.

\section{Recommendations}

- Comprehensive reporting of all actually and potentially relevant patient and disease characteristics that provide scope for determination of predictors of surgical success is prompted.

- Multicenter studies that allow for greater patient populations are encouraged because of disease rarity.

- Well-designed ethically tolerable prospective randomized "control" trials in carefully selected patient population groups should be considered.

- Validated quality of life assessment scales should be incorporated into patients' outcome measures.

\section{Conflict of interest}

The authors Tamer A. EL-Sobky, Shady Samir, Ahmed Naeem Atiyya, Shady Mahmoud, Ahmad S. Aly and Ramy Soliman declare that they have no conflict of interest in connection with this article.

\section{References}

1. Guo XL, Deng Y, Liu HG (2014) Clinical characteristics of hereditary multiple exostoses: a retrospective study of mainland chinese cases in recent 23 years. J Huazhong Univ Sci Technolog Med Sci 34, 42-50. 
2. Jones KB (2011) Glycobiology and the growth plate: current concepts in multiple hereditary exostoses. J Pediatr Orthop 31, 577-586.

3. Masada K, Tsuyuguchi Y, Kawai H, Kawabata H, Noguchi K, Ono K (1989) Operations for forearm deformity caused by multiple osteochondromas. J Bone Joint Surg Br 71, 2429 .

4. Gottschalk HP, Kanauchi Y, Bednar MS, Light TR (2012) Effect of osteochondroma location on forearm deformity in patients with multiple hereditary osteochondromatosis. J Hand Surg Am 37, 2286-2293.

5. Woodside JC, Ganey T, Gaston RG (2015) Multiple osteochondroma of the hand: initial and long-term followup study. Hand. New York, NY, 10, 616-620.

6. Song SH, Lee H, Youssef H, Oh SM, Park JH, Song HR (2013) Modified Ilizarov technique for the treatment of forearm deformities in multiple cartilaginous exostoses: case series and literature review. J Hand Surg Eur 38, 288-296.

7. Litzelmann E, Mazda K, Jehanno P, Brasher C, Pennecot GF, Ilharreborde B (2012) Forearm deformities in hereditary multiple exostosis: clinical and functional results at maturity. J Pediatr Orthop 32, 835-841.

8. Akita S, Murase T, Yonenobu K, Shimada K, Masada K, Yoshikawa H (2007) Long-term results of surgery for forearm deformities in patients with multiple cartilaginous exostoses. J Bone Joint Surg Am 89, 1993-1999.

9. Ishikawa J, Kato H, Fujioka F, Iwasaki N, Suenaga N, Minami A (2007) Tumor location affects the results of simple excision for multiple osteochondromas in the forearm. J Bone Joint Surg Am 89, 1238-1247.

10. Shin EK, Jones NF, Lawrence JF (2006) Treatment of multiple hereditary osteochondromas of the forearm in children: a study of surgical procedures. J Bone Joint Surg Br 88, 255-260.

11. Jiya TU, Pruijs JE, van der Eijken JW (1997) Surgical treatment of wrist deformity in hereditary multiple exostosis. Acta Orthop Belg 63, 256-261.

12. Fogel GRT, McElfresh EC, Peterson HA, Wicklund PT (1984) Management of deformities of the forearm in multiple hereditary osteochondromas. J Bone Joint Surg Am 66A, 670-680.

13. Waters PM, Van Heest AE, Emans J (1997) Acute forearm lengthenings. J Pediatr Orthop 17, 444-449.

14. Refsland S, Kozin SH, Zlotolow DA (2016) Ulnar distraction osteogenesis in the treatment of forearm deformities in children with multiple hereditary exostoses. J Hand Surg Am 41, 888-895.

15. D'Ambrosi R, Barbato A, Caldarini C, Biancardi E, Facchini RM (2016). Gradual ulnar lengthening in children with multiple exostoses and radial head dislocation: results at skeletal maturity. J Child Orthop 10, 127-133.

16. Eralp L, Bilen FE, Rozbruch SR, Kocaoglu M, Hammouda AI (2016) External fixation reconstruction of the residual problems of benign bone tumours. Strategies Trauma Limb Reconstr 11, 37-49.

17. Bauer AS, Storelli DA, Sibbel SE, McCarroll HR, Lattanza LL (2017) Preoperative computer simulation and patientspecific guides are safe and effective to correct forearm deformity in children. J Pediatr Orthop 37, 504-510.

18. Tonogai I, Takahashi M, Tsutsui T, Goto T, Hamada D, Suzue N, Matsuura T, Yasui N, Sairyo K (2015) Forearm lengthening by distraction osteogenesis: a report on 5 limbs in 3 cases. J Med Invest 62, 219-222.

19. Cho YJ, Jung ST (2014) Gradual lengthening of the ulna in patients with multiple hereditary exostoses with a dislocated radial head. Yonsei Med J 55, 178-184.
20. Beutel BG, Klifto CS, Chu A (2014) Timing of forearm deformity correction in a child with multiple hereditary exostosis. Am J Orthop (Belle Mead NJ) 43, 422-425.

21. Vogt B, Tretow HL, Daniilidis K, Wacker S, Buller TC, Henrichs MP, Roedl RW, Schiedel F (2011) Reconstruction of forearm deformity by distraction osteogenesis in children with relative shortening of the ulna due to multiple cartilaginous exostosis. J Pediatr Orthop 31, 393-401.

22. Hill RA, Ibrahim T, Mann HA, Siapkara A (2011) Forearm lengthening by distraction osteogenesis in children: a report of 22 cases. J Bone Joint Surg Br 93, 1550-1555.

23. Bilen FE, Eralp L, Balci HI, Kocaoglu M, Ozger H (2009) Correction of forearm deformities in children with multiple osteochondroma, by corrective radial osteotomy and ulnar lengthening by distraction osteogenesis. Acta Orthop Belg $75,743-747$

24. Matsubara H, Tsuchiya H, Sakurakichi K, Yamashiro T, Watanabe K, Tomita K (2006) Correction and lengthening for deformities of the forearm in multiple cartilaginous exostoses. J Orthop Sci 11, 459-466.

25. Cheng JC (1991) Distraction lengthening of the forearm. J Hand Surg Br 16, 441-445.

26. Pritchett JW (1986) Lengthening the ulna in patients with hereditary multiple exostoses. J Bone Joint Surg Br 68, 561565.

27. Massobrio M, Antonietti G, Pellicano G, Necci F (2015) Single forearm radius and ulna asymmetric lengthening in multiple cartilaginous exostoses: a case report and a longterm follow-up. J Pediatr Orthop B 24, 561-566.

28. Kelly JP, James MA (2016) Radiographic outcomes of hemiepiphyseal stapling for distal radius deformity due to multiple hereditary exostoses. J Pediatr Orthop 36, $42-47$.

29. Arms DM, Strecker WB, Manske PR, Schoenecker PL (1997) Management of forearm deformity in multiple hereditary osteochondromatosis. J Pediatr Orthop 17 450-454.

30. Ip D, Li YH, Chow W, Leong JC (2003) Reconstruction of forearm deformities in multiple cartilaginous exostoses. J Pediatr Orthop B 12, 17-21.

31. Rasool MN (2008) Radioulnar fusion for forearm defects in children - a salvage procedure. SA Orthop J 7 (1), 60-67.

32. Peterson HA (2008) The ulnius: a one-bone forearm in children. J Pediatr Orthop B 17, 95-101.

33. Rodgers WB, Hall JE (1993) One-bone forearm as a salvage procedure for recalcitrant forearm deformity in hereditary multiple exostoses. J Pediatr Orthop 13, 587-591.

34. Yang YF, Zhang GM, Huo ZQ, Xu ZH, Xu DC (2013) Reconstruction of the distal ulnar epiphysis with vascularized proximal fibula including epiphysis in children after osteochondroma resection: report of two cases. Plast Reconstr Surg 132, 784e-789e.

35. Noonan KJ, Levenda A, Snead J, Feinberg JR, Mih A (2002) Evaluation of the forearm in untreated adult subjects with multiple hereditary osteochondromatosis. J Bone Joint Surg Am 84A, 397-403.

36. Moher D, Liberati A, Tetzlaff J. Altman DG, PRISMA Group (2004) (2009) Preferred reporting items for systematic reviews and meta-analyses: the PRISMA statement. BMJ 21(339), b2535.

37. Centre for evidence-based medicine. [Internet]. [cited on 2017 Aug 21]. Available from www.cebm.net/criticalappraisal

38. Slim K, Nini E, Forestier D, Kwiatkowski F, Panis Y, Chipponi J (2003) Methodological index for non-randomized studies (MINORS): development and validation of a new instrument. ANZ J Surg 73, 712-716. 
39. Demir B, Gursu S, Ozturk K, Yildirim T, Konya MN, Er T (2011) Single-stage treatment of complete dislocation of radial head and forearm deformity using distraction osteogenesis in paediatric patients having multiple cartilaginous exostosis. Arch Orthop Trauma Surg 131, 1195-1201.

40. Mader K, Gausepohl T, Pennig D (2003) Shortening and deformity of radius and ulna in children: correction of axis and length by callus distraction. J Pediatr Orthop B 12, 183-191.

41. Tang ZW, Cao YL, Liu T, Chen T, Zhang XS (2013) Management of forearm deformities with ulnar shortening more than $15 \mathrm{~mm}$ caused by hereditary multiple osteochondromas. Eur J Orthop Surg Traumatol 23, 611-618.

42. Burgess RC, Cates H (1993) Deformities of the forearm in patients who have multiple cartilaginous exostosis. J Bone Joint Surg Am 75, 13-18.

43. Irani RN, Petrucelli RC (1993) Ulnar lengthening for negative ulnar variance in hereditary multiple osteochondromas. J Pediatr Orthop B 1, 143-147.

44. Beltrami G, Ristori G, Scoccianti G, Tamburini A, Capanna R (2016) Hereditary multiple exostoses: a review of clinical appearance and metabolic pattern. Clin Cases Miner Bone Metab 13, 110-118.

45. Clement ND, Porter DE (2014) Hereditary multiple exostoses: anatomical distribution and burden of exostoses is dependent upon genotype and gender. Scott Med J 59, $35-44$.
46. Chhina H, Davis JC, Alvarez CM (2012). Health-related quality of life in people with hereditary multiple exostoses. $\mathrm{J}$ Pediatr Orthop 32, 210-214.

47. Darilek S, Wicklund C, Novy D, Scott A, Gambello M, Johnston D, Hecht J (2005) Hereditary multiple exostosis and pain. J Pediatr Orthop 25, 369-376.

48. Pacifici M (2017) Hereditary multiple exostoses: new insights into pathogenesis, clinical complications, and potential treatments. Curr Osteoporos Rep 15, 142-152.

49. Pedrini E, Jennes I, Tremosini M, Milanesi A, Mordenti M, Parra A, Sgariglia F, Zuntini M, Campanacci L, Fabbri N, Pignotti E, Wuyts W, Sangiorgi L (2011) Genotypephenotype correlation study in 529 patients with multiple hereditary exostoses: identification of "protective" and "risk" factors. J Bone Joint Surg Am 93, 2294-2302.

50. Pei Y, Wang Y, Huang W, Hu B, Huang D, Zhou Y, Su P (2010) Novel mutations of EXT1 and EXT2 genes among families and sporadic cases with multiple exostoses. Genet Test Mol Biomarkers 14, 865-872.

51. Jo AR, Jung ST, Kim MS, Oh CS, Min BJ (2017) An evaluation of forearm deformities in hereditary multiple exostoses: factors associated with radial head dislocation and comprehensive classification. J Hand Surg Am 42, 292.e1-292.e8.

52. Sambunjak D, Franić M (2012) Steps in the undertaking of a systematic review in orthopaedic surgery. Int Orthop 36, 477-484.

Cite this article as: EL-Sobky TA, Samir S, Atiyya AN, Mahmoud S, Aly AS, Soliman R (2018) Current paediatric orthopaedic practice in hereditary multiple osteochondromas of the forearm: a systematic review. SICOT-J, 4, 10. 